\title{
Test Reference Text List
}

National Cancer Institute

\section{Source}

National Cancer Institute. Test Reference Text List. NCI Thesaurus. Code C70935.

Textual representation of a normal result of a particular test. It may consist of one word or an expression. 